\title{
RIESGOS PARA EL CONSUMIDOR Y PRODUCTOR EN FUNCIÓN DE LOS CRITERIOS DE ACEPTACIÓN O RECHAZO DE HORMIGÓN PREPARADO SEGÚN DISTINTOS REGLAMENTOS (PARTE II)
}

\author{
RISKS FOR THE READYMIX CONCRETE PRODUCER AND CONTRACTOR \\ ACCORDING TO THE ACCEPTANCE OR REJECTION CRITERIA SPECIFIED \\ IN DIFFERENT STANDARDS (PART II)
}

\author{
D. M. PRADA BETANCOURT(*), D. REVUELTA CRESPO(*), L. FERNÁNDEZ LUCO(*), L. VEGA CATALÁN(*)
}

Recepción/Received: 1-III-05

Aceptación/Accepted: 19-V-05

\section{RESUMEN}

Este trabajo es continuación de la Parte I (1) incluida en este mismo volumen. Sobre la base conceptual de los criterios de conformidad y de aceptación analizados allí, se estudian en este artículo los códigos EN 206-1:2000 (2) y ACI-318 (3) y se compararán ambos con el código EHE (4) analizado en la primera. El trabajo finaliza con unas conclusiones derivadas del análisis precedente.

Palabras clave: hormigón, resistencia a compresión, dispersión, conformidad, criterios de aceptación.
SUMMARY

This paper continues the work done on Part I included in this Volume. Based on the conformity and acceptance criteria analysed on Part I (1), EN 206-1:2000 (2) and ACI-318 (3) standards are studied and compared to EHE (4) standard, described in Part I. The paper conclusions include concepts derived from the previous work.

Keywords: concrete, strength of compression, dispersion, conformity, acceptance criteria.

\section{ANÁLISIS DE LA NORMA EN 206-1:2000 Y DE LAS RECOMENDACIONES DE LA ACI-318}

\section{1. Curvas operativas del criterio de aceptación de la norma europea EN 206-1:2000}

En los códigos EHE y EN 206-1:2000 el criterio de conformidad es el mismo, es decir el hormigón es conforme con la especificación cuando $\theta \leq 5 \%$ y no conforme cuando $\theta>5 \%$, es decir, se fija $\theta_{\text {udm }}=5 \%$. La norma $E N$ 206-1:2000 está orientada hacia el control de producción, exigiendo la calificación de aceptable o rechazable para lotes de hormigón definidos mediante una combinación de criterios temporales y de volúmenes de producción con una capacidad discrecional del productor para delimitar el período de producción sometido a control. La intervención de un organismo tercero independiente también está prevista si lo desea el productor, y tiene como efecto fundamental una disminución de la frecuencia de muestreo y ensayos, pero dicho organismo no define necesariamente el período de producción objeto de control ni tampoco realiza la evaluación del criterio de aceptación, sólo verifica que el fabricante lo hace correctamente.

Existen dos tipos de criterios de aceptación diferentes, uno, aplicable a la llamada producción inicialy otro, a la producción continua o en régimen continuo, siendo

(") Instituto de Ciencias de la Construcción Eduardo Torroja (CSIC), ESPAÑA. Persona de contacto/ Corresponding author. drevuelta@ietcc.csic.es (D. Revuelta Crespo). 
la primera la que opera al inicio de la producción de un nuevo tipo de hormigón y la segunda la que empieza a aplicarse cuando ya se dispone de, al menos, 35 resultados de ensayos.

Muy novedoso es el hecho de que se permite realizar el control sobre familias de hormigones (5), concepto que permite agrupar los resultados - previa transformaciónde varios hormigones individuales en los pertenecientes a un hormigón de referencia que es un miembro destacado de la familia.

La aceptación o rechazo del hormigón de referencia conlleva la aceptación o rechazo de todos los que componen la familia, y está claro que detrás de este procedimiento de agrupación existen razones de economía y de eficiencia técnica que lo justifican. No podemos, por la necesaria brevedad de este trabajo, incidir en este aspecto crucial de la norma EN 206-1:2000, que afecta tanto al control de los hormigones como al propio criterio de aceptación.

La Tabla 1 resume los criterios de aceptación de la norma referida. Nótese en primer lugar que los criterios de aceptación sobre ambos tipos de producción son dobles, al existir, en primer lugar, el llamado Criterio 1 o criterio de la media y, a continuación, un Criterio 2 o criterio individual.

De los cuatro criterios de las otras tantas casillas de la Tabla 1 se va a comentar solamente el Criterio 1, correspondiente a la producción continua. Se aclara, en primer lugar, que la cantidad $\sigma$ que aparece en dicha tabla no es la desviación típica poblacional, sino la desviación típica muestra/ correspondiente a los 35 resultados inmediatamente precedentes al período de control, formado, este último, por la producción, cuyo muestreo ha dado lugar a $\mathrm{n}=15$ resultados muestrales.

La curva operativa de este criterio de aceptación hace intervenir la distribución $t$ de Student no central. A efectos de comparación es suficiente una aproximación a la misma que utiliza la función de distribución $\Phi(\mathrm{z})$ y su inversa $\Phi^{1}(\mathrm{z})$. Tal aproximación es - véase López Agüi (6)-.

$$
P_{A}(\theta) \cong \Phi\left[\frac{-\Phi^{-1}(\theta)-1.469}{0.314}\right]
$$

cuya gráfica aparece en la Figura 1.

Nótese que el riesgo del consumidor es

$$
\beta=P_{\Lambda}(0.05) \cong \Phi(0.56)=0.7124
$$

A diferencia del criterio de aceptación de la EHE, ahora la curva operativa es única y se pueden calcular las probabilidades que se deseen sin necesidad de conocer ningún parámetro poblacional -recuérdese que en el caso de la EHE era necesario conocer el coeficiente de variación poblacional-.

\subsection{Curvas operativas del criterio de aceptación en las recomendaciones de la ACI-318}

Las recomendaciones de la ACI-318 están orientadas a conseguir un estándar de producción, suficientemente controlado, que permita confiar en un suministro de hormigón a una obra concreta con la calidad requerida.

Utilizando la misma terminología que en los apartados anteriores, el estándar de producción se establece mediante la definición de una resistencia media requerida dada por la expresión:

$$
\mu_{r e q}=\operatorname{máx}\left\{\mu_{r e q, l} ; \mu_{r e q q^{2}}\right\}
$$

siendo:

$$
\begin{aligned}
& \mu_{r e q q^{\prime}{ }^{\prime}}=f_{c k}+1.34 \sigma \\
& \mu_{r e q^{\prime} 2}=f_{c k}+2.33 \sigma-3.5
\end{aligned}
$$

y $\sigma$ la desviación típica de la población calculada a partir de datos históricos según el procedimiento fijado en las propias recomendaciones $\mathrm{ACI}$.

La resistencia media requerida, $\mu_{\text {req, }}$ es la base para definir la dosificación del hormigón a suministrar. En efecto, la resistencia media real finalmente adoptada por el fabricante como consigna de fabricación, designada por $\mu$, deberá ser mayor o igual que $\mu_{\text {req }}$ para que la producción cumpla las recomendaciones de la ACI.

Es claro que la dependencia de $\mu_{\text {req }}$ respecto de $\sigma$ hace que las modificaciones de este último parámetro poblacional deban reflejarse inmediatamente en $\mu_{\text {req }}$ a través de la Ec. [4] y la Ec. [5], y, por ende, en $\mu_{\text {req, a }}$ través de la Ec. [3]. Es útil calcular el valor de $\sigma$ para el que $\mu_{\text {req }} 1=\mu_{\text {req }} 2$. Igualando la Ec.[4] y la Ec. [5], se deduce inmediatamente que el valor separador de la desviación típica, designado por $\sigma_{0}$, vale $\sigma_{0}=3,53 \mathrm{MPa}$. Para $\sigma=\sigma_{0}$ es $\mu_{\text {req }}=\mu_{\text {req }} 1$, mientras que para $\sigma>\sigma_{0}$ es $\mu_{\text {req }}=\mu_{\text {req }} 2$.

La Figura 2 muestra la evolución de mreq con $s$ mediante la línea quebrada $A B C$. La conformidad de la producción con $A C I$ requiere que $\mu=\mu_{\text {req }}$, y por tanto todos los puntos situados en la línea quebrada $A B C$ o por encima de ella corresponden a producciones -o dosificaciones- conformes con $\mathrm{ACI}$, mientras que los puntos situados por debajo representan producciones no conformes con ACI.

También se ha trazado en la figura la línea recta $A D$, de ecuación $\mu=\mathrm{f}_{c k}+1.64 \sigma$ que juega el mismo papel que $A B C$ en relación con los códigos EHE y EN 2061: 2000. Los puntos situados en o por encima de la línea $A D$ son conformes con EHE y EN 206-1 mientras que son no conformes los situados por debajo. El punto $E$ en que se cruzan ambas líneas se calcula fácilmente haciendo que $\mu_{\text {req }} 2$ sea igual a la $f_{c k}+1.64 \sigma$. Designando por $\sigma_{1}$ a 
TABLA 1

Criterios de aceptación para la resistencia en la EN 206-1:2000

\begin{tabular}{|c|c|c|c|}
\hline PRODUCCIÓN & $n$ & Criterio 1 & Criterio 2 \\
\cline { 3 - 4 } & & $\begin{array}{c}\text { Media de } \mathrm{n} \text { resultados } \\
\bar{X}_{n} \text { en } \mathrm{N} / \mathrm{mm}^{2}\end{array}$ & $\begin{array}{c}\text { Resultados individual } \\
X_{i} \text { en N/mm }\end{array}$ \\
\hline Inicial & 3 & $\geq f_{c k}+4.0$ & $\geq f_{c k}-4.0$ \\
\hline Continua & 15 & $\geq f_{c k}+1.48 \sigma$ & $\geq f_{c k}-4.0$ \\
\hline
\end{tabular}

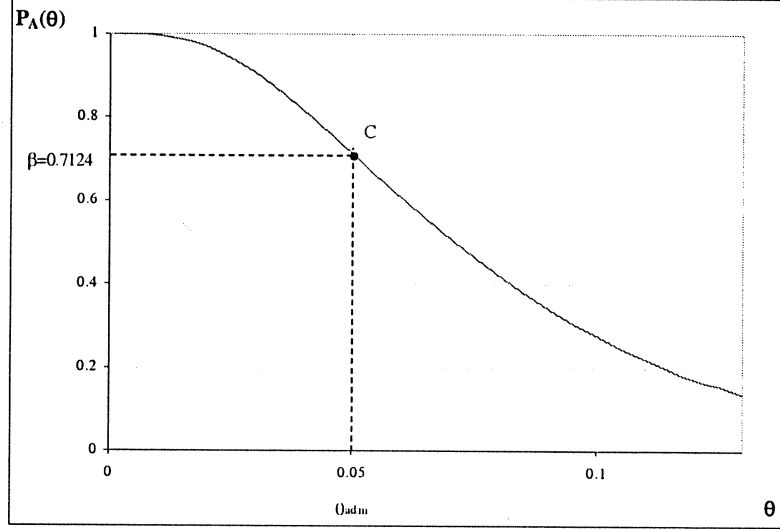

Figura 1.- Curva operativa del Criterio 1 y producción continua de la EN 206-1.

este nuevo valor separador se obtiene $\sigma_{1}=5,07 \mathrm{MPa}$. Para producciones con $\sigma<\sigma_{1}=5,07 \mathrm{MPa}$ los códigos EHE y EN 2061 son más exigentes que el código ACI a la hora de establecer la conformidad. Así, la producción representada por $\mathrm{F}$ es conforme para el código ACI y no lo es para EHE y EN 2061. En cambio para producciones con $\sigma>\sigma_{1}=5,07 \mathrm{MPa}$ sucede lo contrario. En este caso, la producción representada por $\mathrm{G}$ es conforme para EHE y EN 2061 y no conforme para ACI. Podría decirse que en la práctica de la producción, en países con tecnología de fabricación media-alta y para hormigones de resistencia especificada $f_{f=}=50 \mathrm{MPa}$, desviaciones típicas $\sigma<3,4 \mathrm{MPa}$ corresponden a producciones con dispersión baja, desviaciones 3,5 $\sigma \quad 6,0 \mathrm{MPa}$ a producciones con dispersión media y desviaciones con $\sigma>6.0$ $\mathrm{MPa}$ a producciones con dispersión alta. El hecho de que los códigos EHE y EN 2061 sean neutrales respecto de la dispersión a la hora de establecer el criterio de conformidad - no confundir con el criterio de aceptación- mientras que el código ACI favorezca las bajas dispersiones y castigue las altas en este capítulo, nos parece un acierto de este último, pues las fracciones subnominales se comportan de forma muy diferente - con las consecuencias que de ello se derivan para la seguridad estructural- según sea la dispersión poblacional subyacente. Dicha figura puede usarse también con el propósito de representar las fracciones subnominales efectivas que corresponden a cada producción -es decir a cada punto del gráfico-. A tal efecto, la ecuación de la fracción subnominal $\theta$ en función

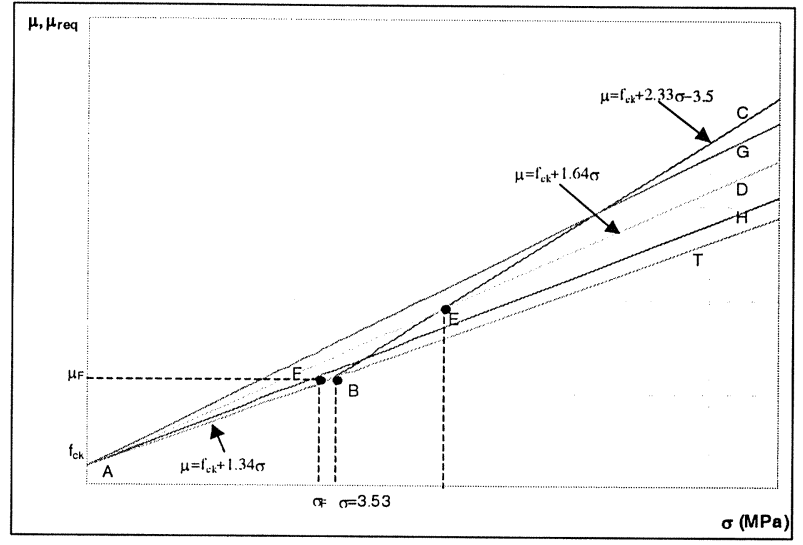

Figura 2.- Zonas de conformidad con EHE, ENE 206:2000 y ACI318.

de la resistencia característica nominal, $f_{c k^{\prime}}$ que se describía en la Parte I de este trabajo,

$$
\theta=\Phi\left(\frac{f_{c k}-\mu}{\sigma}\right)=1-\Phi\left(\frac{\mu-f_{c k}}{\sigma}\right)
$$

también se puede expresar de la forma:

$$
\mu=f_{c k}-\Phi^{\prime}(\theta) \sigma
$$

lo que demuestra que considerando $\theta$ como parámetro, la Ec. [7] representa un haz de rectas de vértice $A$ y pendiente $-\Phi^{-1}(\theta)$. Resulta, pues, que los puntos - producciones- de una recta que pase por $A$, como por ejemplo la recta $A H$, representan producciones diferentes pero todas ellas con la misma fracción subnominal. Para calcularla basta elegir cualquier punto de la recta diferente de A y aplicar la Ec. [6]. Por ejemplo, eligiendo $F$ se tiene que todas las producciones de la recta $\mathrm{AH}$ comparten la fracción subnominal $\theta_{A H}=\Phi\left[\left(f_{c k}-\mu_{F}\right) / \sigma_{F}\right]$.

A la recta $A D$, corresponde la fracción subnominal $\theta \mathrm{AH}=\Phi(-1,64)=0,05, \mathrm{y}$, por ello, el criterio de conformidad en los códigos EHE y ENE 206-1 se puede establecer también en la forma $\theta \leq \theta_{\text {adm }}$ con 
$\theta_{\text {adm }}=0,05$, pues resulta equivalente exigir que $\mu>$ $\mathrm{f}_{\mathrm{ck}}+1.64 \sigma$ o que $\theta<\theta$ adm .

En el código ACI, por el contrario, debe tenerse cuidado a la hora de transformar el criterio de conformidad $\mu \geq \mathrm{m}$ req en otro que utilice fracciones subnominales. El hecho de que la frontera de la zona de conformidad sea la línea quebrada $A B C$ obliga a establecer la fracción subnominal admisible, $\theta_{\mathrm{adm}}$ diferenciando el tramo $A B$ del tramo $B C$.

Como el segmento $A B$ pertenece a la recta $A T$ del haz que tiene por foco el punto $A$, los puntos de $A B$ tienen la misma fracción subnominal, que es, a su vez, fracción subnominal admisible por estar en la frontera de la zona de conformidad.

Apoyándonos en el punto B podemos calcular inmediatamente dicha fracción subnominal:

$$
\theta_{A B}=\Phi\left(\frac{f_{c k}-\mu_{B}}{\sigma_{B}}\right)=\Phi(-1,34) \cong 0.0901=9.01 \%
$$

En cambio en el tramo BC a cada punto corresponde una fracción subnominal diferente porque la recta $B C$ no pasa por $A$.

Esta fracción subnominal es función de la desviación típica correspondiente a cada producción o punto. Como en todos los puntos de dicha recta se verifica que $\mu=f_{c k}$ $+2.33 \sigma-3.5$, de la Ec. [6] se deduce:

$$
\theta_{E C}=\left(\frac{f_{c k}-\mu}{\sigma}\right)=\Phi\left(-2.33+\frac{3.5}{\sigma}\right)
$$

En consecuencia, la fracción subnominal admisible implícita en la definición de conformidad del código ACI viene dada por:

$$
\theta_{a d m}= \begin{cases}0.0901 & ; \sigma \leq 3.54 \mathrm{MPa} \\ \Phi\left(-2.33+\frac{3.5}{\sigma}\right) & ; \sigma>3.54 \mathrm{MPa}\end{cases}
$$

Con esta función se obtiene fácilmente la Tabla 2.

Puede verse que la fracción subnominal admisible, $\theta$ adm, varía inversamente con la desviación típica. Esta evolución se considera razonable y congruente con el concepto de seguridad teniendo en cuenta que los resultados subnominales de la resistencia se agrupan más cerca de $f_{c k}$ cuanto menor sea la desviación típica.
La ACI318 establece las siguientes pautas muestrales: "las muestras para los ensayos de resistencia de cada clase de hormigón colocado cada día deben tomarse no menos de una vez al día, ni menos de una vez cada $120 \mathrm{~m}^{3}$ de hormigón, ni menos de una vez cada $500 \mathrm{~m}^{2}$ de superficies de losas y muros." Aunque no está claro el concepto de "clase de hormigón", se puede suponer la clasificación del hormigón de acuerdo a su consistencia, sin embargo se considera inconveniente que este término quede bajo la interpretación del usuario.

La aceptación de un hormigón en obra se basa en dos requisitos que se deben cumplir simultáneamente: "Cada promedio aritmético de tres ensayos de resistencia consecutivos es igual o superior a $f$ " $y$ "ningún resultado individual del ensayo de resistenćla (promedio de dos probetas) debe ser menor que $f$ menos 3,5 $\mathrm{MPa}$ ". Con respecto al primer requisito cabbe aclarar que no se especifica si el cálculo del promedio se refiere a agrupamientos de los resultados consecutivos con solapamiento o sin solapamiento. En este trabajo se realizaron pruebas de las dos maneras y se detectó que si el promedio con solapamiento no cumple lo establecido por la norma, esta deficiencia se observa con mayor impacto debido a que, por lo menos, tres promedios consecutivos fallarían el mínimo establecido, porque el criterio de aceptación se aplica con una frecuencia triple. De igual manera se observó que cuando el promedio se realizaba sin solapamiento, el error se presentaba sólo en un cálculo de promedio, es decir, de forma menos evidente, aunque en ambos casos con análoga frecuencia relativa.

Con la ayuda del cálculo de probabilidades se concluye que las ecuaciones analíticas de las curvas operativas para los dos criterios de aceptación de la ACI318 son:

Criterio 1: $\quad P_{A}(\theta)=\Phi\left[-\sqrt{3} \Phi^{-1}(\theta)\right]$

Criterio 2: $\quad P_{A}(\theta)=\left[\Phi\left(\frac{3.5}{\sigma}\right)-\Phi^{-1}(\theta)\right]^{3}$

Partiendo de estas dos ecuaciones, se obtienen las dos curvas operativas para cada uno de los criterios (Figura 3). Obsérvese que la probabilidad de aceptación del primer criterio depende sólo de la fracción defectuosa, mientras que para el segundo criterio depende de la fracción defectuosa y de la desviación estándar de la producción. Por esto se presentan curvas operativas para diferentes desviaciones estándar.

\section{CONCLUSIONES}

Los códigos analizados contemplan de forma diferente los criterios de conformidad y de aceptación. EHE y EN 2061:2000 refieren la conformidad fijando un valor constante para la máxima fracción subnominal admisible, 
TABLA 2

Valores de $\mathrm{q}_{\mathrm{adm}}$ en función de $\mathrm{s}$ en las recomendaciones de la ACI-318

\begin{tabular}{|l|c|c|c|c|c|c|c|}
\hline$\sigma(\mathrm{MPa})$ & 3.0 & 3.5 & 4.0 & 5.0 & 6.0 & 7.0 & 8.0 \\
\hline$\theta_{a d m}(\%)$ & 9.01 & 9.01 & 7.28 & 5.16 & 4.03 & 3.36 & 2.92 \\
\hline
\end{tabular}
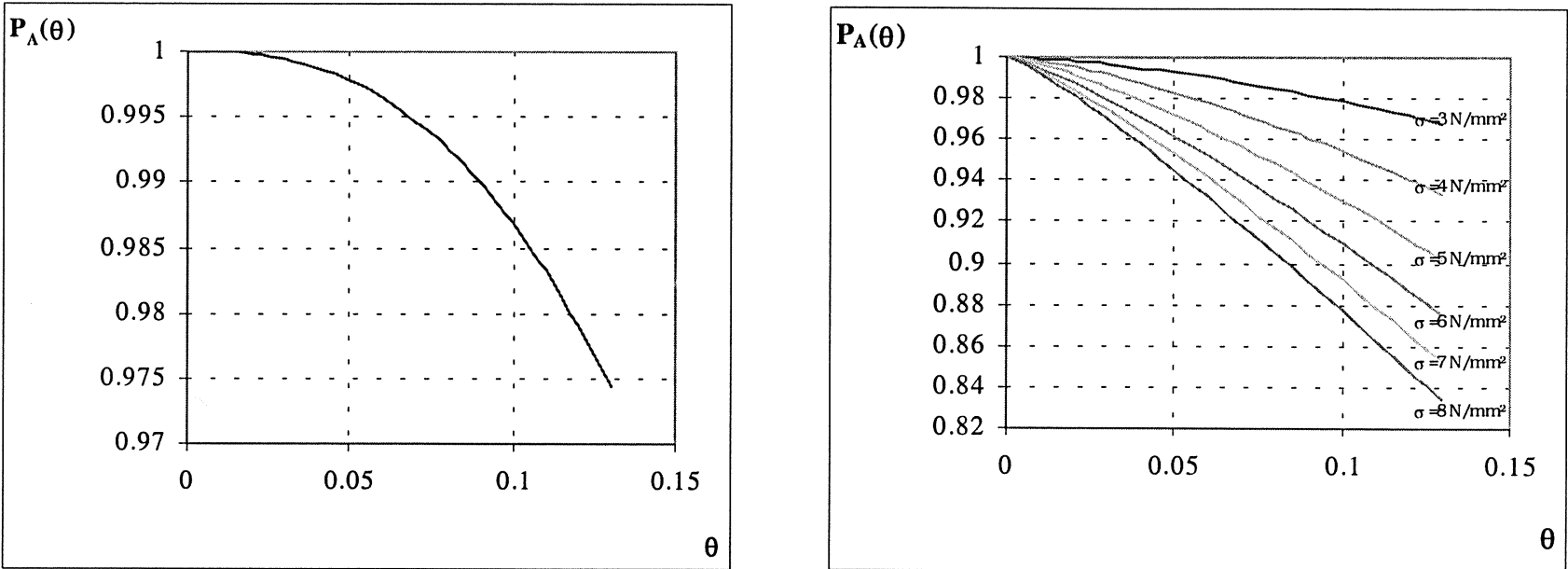

Figura 3.- Curvas operativas Criterios 1 y 2, ACI-318.

$\theta_{\text {adm }}$ o calidad límite. Por el contrario la ACI138, establece implícitamente una fracción subnominal admisible inversamente variable con la desviación típica, lo que se considera más adecuado desde el punto de vista de la seguridad estructural.

Los criterios de aceptación son difícilmente comparables, tanto porque los tamaños muestrales son diferentes como porque el control utiliza diferentes perspectivas: Control de producción en la EN 2061:2000, control de recepción * en la EHE y mixto en la ACI. Los riesgos del consumidor pueden parecer algo elevados en el caso de ACI y de EHE en segunda etapa. No obstante, este parámetro resulta secundario en el planteamiento del código ACI si se consigue mediante el control de producción oportuno que la resistencia media requerida sea respetada. Éste es el verdadero parámetro poblacional a controlar. El criterio de aceptación, en tal caso, tiene la misión de asegurar riesgos moderados al fabricante que opera con la calidad adecuada.

\section{AGRADECIMIENTOS}

Los autores del presente trabajo agradecen al Dr. Ingeniero Juan Carlos Lopez Agüi, Director General de IECA-Instituto Español del Cemento y sus Aplicaciones, la colaboración prestada para la realización del mismo.

\section{BIBLIOGRAFÍA}

(1) D. M. Prada Betancourt, D. Revuelta Crespo, L. Fernández Luco, L. Vega Catalán, «Riesgos para el consumidor y productor en función de los criterios de aceptación o rechazo de hormigón preparado según distintos reglamentos-Parte I», Mater. Construcc., Vol. $55, n^{\circ} 280$ (2005), pp. 71-77.

(2) Comisión Europea de Normalizacion, CEN, European Standard EN 206-1, 2000.

(3) ACI Committee 318, Building Code Requirements for Structural Concrete, 2002.

(4) Comisión Permanente del Hormigón, EHE, Instrucción para el proyecto y la ejecución de obras de hormigón en masa o armado, MFOM, 1998.

(5) T. A. Harrison, The Use of Concrete Families for the Production and Conformity Control of concrete, 1999.

(6) J. C. López Agüi et al., Control estadístico del hormigón estructural, M-7. ACHE, 2003. 